\title{
Localized surface plasmon resonance modulated double F-P resonance cavities to improve the absorption of perovskite solar cells
}

\author{
Chun-Ping Xiang ${ }^{1}$, Yu Jin ${ }^{2}$ \\ ${ }^{1}$ College of Information Engineering, Jimei University \\ ${ }^{2}$ Fujian Key Laboratory of Light Propagation and Transformation, College of Information Science and Engineering, Huaqiao \\ University \\ No. 183, Yinjiang Road, Jimei District \\ Xiamen, China \\ Tel: +86 18064518060 Fax: +86 0592-6181378 E-mail: chunpingxiang@jmu.edu.cn
}

\begin{abstract}
Organometal lead halide perovskites were identified as promising absorbers for thin-film solar cells. To improve the absorption of perovskite solar cells (PSCs), silica nano-rods were introduced into perovskite layer of PSCs. We characterized the coupled modes between dipole localized surface plasmon resonance (LSPR) and Fabry-Pérot (F-P) resonance. By optimizing the structure of PSCs, the double F-P resonances occurred at wavelength of $\sim 590 \mathrm{~nm}$ and $\sim 780 \mathrm{~nm}$ simultaneously.
\end{abstract}

Key words: Perovskite solar cells, Localized surface plasmon resonance and Double F-P resonance

\section{Introduction}

Solar cells with high power conversion efficiency (PCE), low cost, long-term application reliability and environmental friendly performance become the focus of current energy research[1]. Thin-film photovoltaics based on hybrid metal-organic semiconductor materials play an important role in the field of clean renewable energy[2]. Perovskite solar cells (PSCs) which use the organometal methylammonium lead halide material as the light absorber have attracted great attention in photovoltaic research in recent years, due to their superb PCE as well as low material costs[3]. The PCE of PSCs as high as $22.1 \%$ has been achieved in 2017 since the first report on PSCs which showed a PCE of $3.8 \%[4,5]$. The high photovoltaic performance of perovskites is attributed to both the optically high extinction coefficients together with high internal quantum efficiency and the electrical balanced charge carriers mobilities as well as diffusion lengths[6]. The general molecular formula of organometal perovskite used as the absorber of PSCs is $\mathrm{ABX}_{3}$, where $\mathrm{A}$ represents the organic cation (e.g. $\mathrm{CH}_{3} \mathrm{NH}_{3}{ }^{+}, \mathrm{NH}_{2} \mathrm{CH}=\mathrm{NH}_{2}{ }^{+}, \mathrm{CH}_{3} \mathrm{CH}_{2} \mathrm{NH}_{3}{ }^{+}$), $\mathrm{B}$ is the metal cation (e.g. $\mathrm{Pb}^{2+}, \mathrm{Sn}^{2+}, \mathrm{Ge}^{2+}$ ) and $\mathrm{X}$ indicates the halogen anion (i.e. $\left.\mathrm{F}^{-}, \mathrm{Cl}^{-}, \mathrm{Br}^{-}, \mathrm{I}^{-}\right)[3]$. As a direct bandgap semiconducting pigment, $\mathrm{CH}_{3} \mathrm{NH}_{3} \mathrm{PbI}_{3}$ has been widely used in developing low-cost PSCs[2]. The intrinsic absorption of $\mathrm{CH}$ ${ }_{3} \mathrm{NH}_{3} \mathrm{PbI}_{3}$ locates in wavelength smaller than $800 \mathrm{~nm}$ due to a bandgap of $1.55 \mathrm{eV}$. The excitons' binding energy of $\mathrm{CH}-$ ${ }_{3} \mathrm{NH}_{3} \mathrm{PbI}_{3}$ produced by light absorption is about $0.03 \mathrm{eV}$, resulting in a fast dissociation of excitons at room temperature[7]. The high carrier mobilities $\left(7.5 \mathrm{~cm}^{2} \mathrm{~V}^{-1} \mathrm{~s}^{-1}\right.$ for electrons and $12.5 \mathrm{~cm}^{2} \mathrm{~V}^{-1} \mathrm{~s}^{-1}-66 \mathrm{~cm}^{2} \mathrm{~V}^{-1} \mathrm{~s}^{-1}$ for holes) together with long carrier-diffusion lengths $(\sim 1$ um for electrons and $\sim 1.2$ um for holes) make it possible to get a high carrier collection efficiency in PSCs[8-10].

Various of typical structures of PSCs including the mesoporous scaffold, the bulk heterojunction and the (inverted) planar heterojunction have been reported with excellent performance in previous research[1, 11]. For the planar heterojunction PSCs, the hole transport layer (HTL) together with perovskite absorbing layer and electron transport layer (ETL) constitute the planar p-i-n heterojunction part, which is sandwiched by cathode and anode. The structure forms a Fabry-Pérot (F-P) resonance microcavity which has been widely used for light trapping in thin-film solar cells[12]. The $\mathrm{PC}_{61} \mathrm{BM}((6,6)$-phenyl-C61-butyric acid methyl ester) film acts as ETL, $\mathrm{Au}$ or $\mathrm{Ag}$ film is selected as cathode and the transparent conductive oxide (e.g. ITO or TCO) acts as anode $[6,13]$. Instead of liquid electrolyte, the solid-state HTL (e.g.

spiro-MeOTAD $\left(2,2^{\prime}, 7,7^{\prime}\right.$-tetrakis-( $N, N$-di-p-methoxyphenylamine $) 9,9^{\prime}$-spirobi fluorene) polyTPD (poly( $N, N^{\prime}$-bis(4-n-butylphenyl)- $N, N^{\prime}$-bis(phenyl)benzidine)) has been introduced into PSCs and achieve a superb PCE as well as cell stability[14]. As light incidents, the free charge carriers generate in perovskite layer. They diffuse and drift under the effect of the internal electric field due to different energy band levels of materials: electrons moving toward the ETL while holes moving toward the HTL. In order to achieve a higher light absorption, the thickness of perovskite layer is usually set to $200 \mathrm{~nm} \sim 900 \mathrm{~nm}$ and to satisfy the F-P resonance condition[15]. However, the quality of perovskite film becomes compromised with the increase of thickness due to shorting defects arising from large crystals[15]. A thick perovskite film leads to an increase of recombination which resulting in loss in device performance, such as current density and fill factor[6]. Thus, the thickness of perovskite layer is usually less than $550 \mathrm{~nm}$. Moreover, the high extinction coefficient $(\mathrm{k})$ of perovskite materials makes the intensity of light decreases exponentially throughout the active layer and the decay length is less than $150 \mathrm{~nm}$ in optical absorbing spectrum[12]. Thus, the intensity of light at the rear side is weaker than that at the front side of perovskite layer.

The localized field enhancement effect of localized surface plasmon resonance (LSPR) is widely used to improve solar energy harvesting in thin-film photovoltaics[16]. Meanwhile, nanostructures show light trapping effect in solar cells resulting in an improvement of efficiency. A planar hetrojunction structure PSCs containing multilayers complicate the modulation of light field because of the F-P cavity resonance formed in device will couple with other optical modes (e.g. LSPR, waveguide modes). In the current work, we analyze the 
modes coupling between dipole LSPR and F-P resonance and design a double F-P resonance cavity by inducing silica nano-rods. Numerical study based on Rigorous Coupled Wave Analysis (RCWA) method for the whole model calculation and Finite-Difference Time-Domain (FDTD) method for the calculation of absorption in separated layers in a one-dimensional nano-structure device show an improvement on light harvest.

\section{Simulation model}

Based on a typical inverted heterojunction planar or structured PSCs, the schematic of PSCs with a one-dimensional nano-rods cross section is shown in Fig. 1(a). ITO (95 nm) /PEDOT:PSS (70 $\mathrm{nm}) \quad$ (indium tin oxide/poly(3,4-ethylenedioxythiophene):poly(styrene sulfonate)) prepared on transparent glass substrate act as the anode, the polyTPD $(10 \mathrm{~nm}) / \mathrm{CH}_{3} \mathrm{NH}_{3} \mathrm{PbI}_{3} / \mathrm{PC}_{61} \mathrm{BM}(10 \mathrm{~nm})$ forms the p-i-n heterojunction, Au cathode film (300 nm) will reflect the incident light. The silica nano-rods are embedded at the rear side of perovskite layer. The thickness of active layer, the period of nano-rods and the distance between nano-rods and PC61BM layer are adjustable parameters. Firstly, two ideal models of $\mathrm{Au} / \mathrm{PC}_{61} \mathrm{BM} /$ perovskite interface and PSCs are investigated to analyze LSPR, F-P resonance and coupling between them. In the ideal model, the extinction coefficient of all nonmetallic materials is set to zero to get rid of the absorption of organic materials. Moreover, based on the structure of the ideal model, a real model of PSCs which taking the whole absorption into account is simulated to quantify the improvement of intrinsic absorption. In our simulation, the transverse magnetic (TM) -polarized light source incidents normally, the perfectly matched layer (PML) absorbing boundary condition is applied along the direction of propagation of light in our simulation box while in the lateral directions, the periodic boundary condition is implemented and the wide of simulation box define the period of nano-rods. The optical constants (i.e. $\mathrm{n}$ and $\mathrm{k}$ ) of all materials are achieved from literatures[6, 17-19].

Fig. 1(b) shows the optical constant of $\mathrm{CH}_{3} \mathrm{NH}_{3} \mathrm{PbI}_{3}$. Two sharp edges locating at $500 \mathrm{~nm}$ and $800 \mathrm{~nm}$ can be found in $\mathrm{k}$ (blue) curve. For wavelength in region of $500 \mathrm{~nm} \sim 800 \mathrm{~nm}$, the optical field is significantly affected by the structure of micro-cavity formed in PSCs, resulting in F-P resonance. (a)

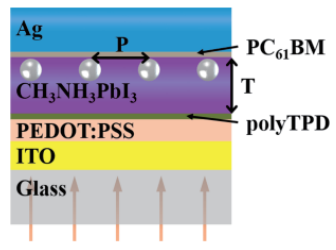

(b)

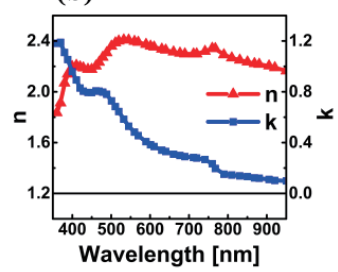

Fig. 1 (a) Schematic of PSCs. (b) Optical constants of $\mathrm{CH}_{3} \mathrm{NH}_{3} \mathrm{PbI}_{3}$

\section{Results discussion}

\section{A. Resonance in ideal model}

Initially, we calculate the absorption profile of an ideal planar cavity model and of an ideal interface model by the
RCWA method in order to analyze the LSPR and F-P resonance. A planar cavity can satisfy the F-P resonance conditionally, which is expressed as:

$$
\mathrm{n}_{a} d_{a}=\frac{c \cdot[\pi \cdot m-\Psi(f)]}{2 \cdot \pi \cdot f \cdot \cos (\theta)}
$$

where $n_{a}$ and $d_{a}$ is the refractive coefficient and thickness of active layer, respectively, $\psi(f)$ describes the frequency-dependent phase shift at the interface, $\mathrm{m}$ represents the order of F-P mode[20]. The absorption spectra of ideal planar PSCs structure without nano-rods are shown in Fig. 2(a). The F-P resonance red-shifts with the increase of the thickness of cavity and affected by the optical constant of $\mathrm{Au}$ significantly. The frequency of F-P resonance is a function of parameter $\mathrm{m}$. In the cavity interference region, only one wavelength can satisfy the F-P resonance condition for the device with a fixed thickness of active layer, resulting in a limit of the broad spectra absorption enhance.
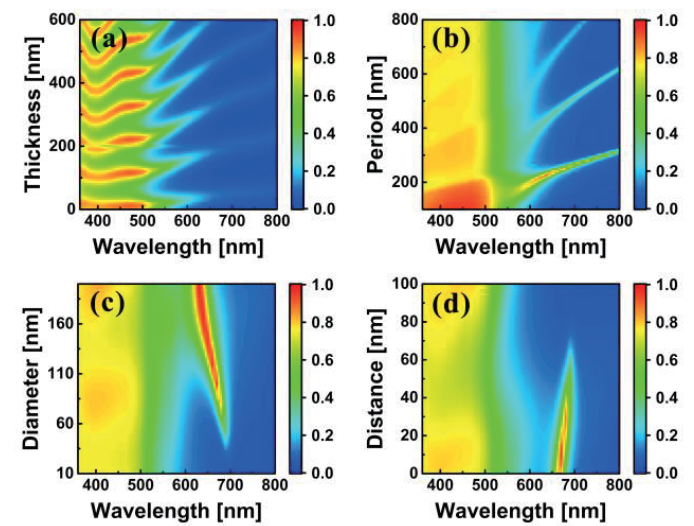

Fig. 2 (a) Absorption spectra of an ideal planar structured PSCs as a function of thickness of perovskite. Absorption spectra of an ideal $\mathrm{Au} /$ $\mathrm{PC}_{61} \mathrm{BM} /$ perovskite interface as a function of period of nano-rods (b), diameter of nano-rod (c) and distance between $\mathrm{PC}_{61} \mathrm{BM} /$ perovskite interface and silica nano-rods (d). The default parameter of period, diameter and distance is set to $250 \mathrm{~nm}, 100 \mathrm{~nm}$ and $10 \mathrm{~nm}$, respectively.

An ideal $\mathrm{Au} / \mathrm{PC}_{61} \mathrm{BM} /$ perovskite interface embedded with silica nano-rods in perovskite layer shows a dipole LSPR character since the refractive index of silica is lower than that of perovskite[21]. Fig. 2(b), (c) and (d) shows the simulation result of the ideal interface. The resonance around silica nano-rods localizes the light field and enhances the absorption of $\mathrm{Au}$ at wavelength larger than $500 \mathrm{~nm}$. The dipole resonance red-shifts with the increase of period and the intensity decreases in higher resonance order. As the diameter of nano-rod increases, the resonance blue-shifts and shows stronger absorption enhancement. It can be seen that nano-rod with diameter lower than $30 \mathrm{~nm}$ shows little enhance effect. Fig. 2(d) indicates that the dipole resonance shows a strong distance dependence. The dipole LSPR decreases with the increase of distance and performs a weak enhancement when the distance larger than $40 \mathrm{~nm}$. Thus, in order to achieve an effective nano-rod-induced resonance, the nano-rod should set the diameter larger than $30 \mathrm{~nm}$ and close to Au interface.

\section{B. Coupled modes}


The nano-rods are introduced in the ideal planar PSCs model to analyze the coupled modes between F-P resonance and dipole LSPR, schematic as Fig. 1(a) shows. Fig. 3 shows the absorption spectra as a function of thickness in the real structured PSCs model. The dipole LSPR induced by nano-rods coupled with the F-P resonance in wavelength region of $500 \mathrm{~nm} \sim 800 \mathrm{~nm}$ when the period of nano-rods ranges from $150 \mathrm{~nm}$ to $300 \mathrm{~nm}$. The coupled modes enhance the absorption of $\mathrm{Au}$ significantly [point $\mathrm{a}$ in Fig. 3(a)]. Moreover, the increases of the diameter of nano-rod will broaden the spectra's width of coupled modes. It is noticed in Fig. 3 that the coupled modes expend along the vertical direction, indicating that these coupled modes have large thickness compatibility. Especially, for the thickness of perovskite closes to the diameter of nano-rods, the F-P resonance will strongly interfere with the dipole LSPR, the hybrid plasmon-cavity polaritons are excited [point $b$ in Fig. 3(b)][20]. The grating-induced waveguide modes generate at wavelength region smaller than dipole resonance. They show a narrow absorption peak, which limits their ability of absorption enhancement [point $\mathrm{c}$ in Fig. $3(\mathrm{~b})][22]$.
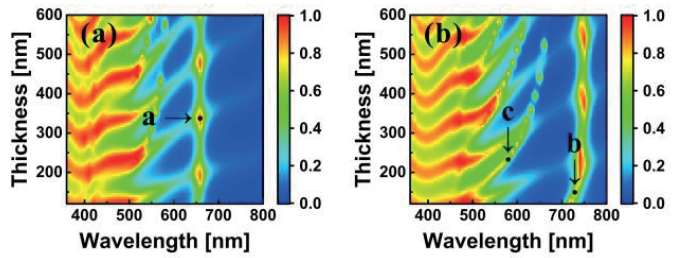

Fig. 3 Absorption spectra of ideal PSCs model with nano-rods embedded at the rear side of perovskite layer. The period and diameter of nano-rods is set to $250 \mathrm{~nm}$ and $80 \mathrm{~nm}$ for (a) and $300 \mathrm{~nm}$ and 100 $\mathrm{nm}$ for (b), respectively.

\section{Absorption enhancement}

In order to demonstrate the intrinsic absorption improvement of perovskite layer, a real model of PSCs structure with nano-rods embedded is calculated by the FDTD method. As Fig. 4 shows, the absorption of perovskite in wavelength region of $550 \mathrm{~nm} \sim 800 \mathrm{~nm}$ is affected by the F-P resonance. For a planar device without nano-rods, only one order F-P resonance can locate in the intrinsic absorption region. When 70 $\mathrm{nm}$-diameter nano-rods are introduced in perovskite layer, the intrinsic absorption at wavelength of $780 \mathrm{~nm}$ improves due to the coupled modes raised, meanwhile, the F-P resonance blue-shifts. A larger diameter nano-rod will blue-shifts coupled modes and weaken the F-P resonance at wavelength around $600 \mathrm{~nm}$, by comparison, nano-rods with diameter set to $20 \mathrm{~nm}$ show little enhancement. It is pointed out that the effective thickness of perovskite layer decreases for PSCs with nano-rods embedded comparing with PSCs without nano-rods. Perovskite layer performs higher absorption efficiency with smaller effective thickness in the optimized PSCs device. In order to demonstrate the modulation effect, we also calculate the absorption spectra distribution in perovskite layer in planar device and show it in the inset of Fig. 4. It can be seen that the light with wavelength smaller than $500 \mathrm{~nm}$ is fully absorbed at the front side of perovskite layer. The F-P (or quasi F-P) resonance character is found in both planar devices with/without nano-rods. It is noticed that the cavity with $70 \mathrm{~nm}$ diameter nano-rods embedded at the rear side of perovskite layer shows double F-P resonance character at $\sim 590 \mathrm{~nm}$ and $\sim$ $780 \mathrm{~nm}$ and improves the intrinsic absorption.

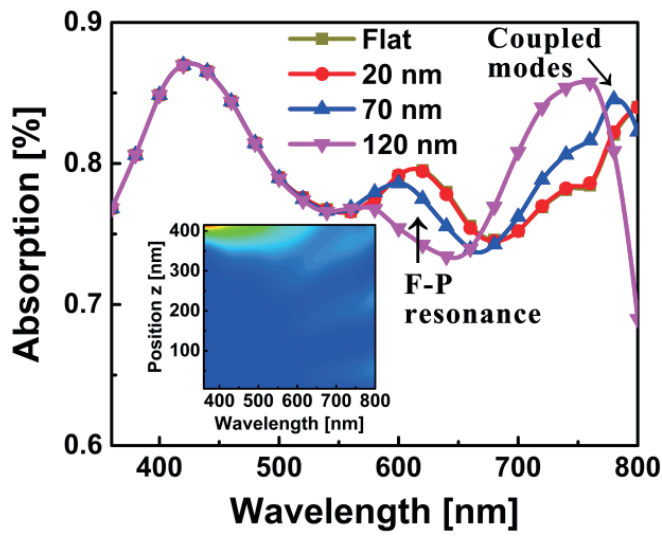

Fig. 4 Absorption spectra of perovskite layer in real model with different diameters of nano-rods. Thickness of perovskite layer, period of nano-rods and distance between nano-rods and Au are set to $420 \mathrm{~nm}, 200 \mathrm{~nm}$ and $10 \mathrm{~nm}$, respectively. The inset is the absorption spectra distribution in perovskite layer of planar PSCs model.

\section{Conclusion}

In this work, the coupled modes between dipole LSPR and F-P resonance is investigated in ideal PSCs model embedded with nano-rods at the rear side of perovskite layer in order to improve the absorption of perovskite. The absorption enhancement of perovskite material is simulated in real PSCs structure. The coupled modes red-shift (blue-shift) with the increase of period (diameter) of nano-rods, meanwhile, the dipole LSPR shows large nano-rods' period dependence. The enhanced electric field around nano-rods improves the absorption of perovskite. For an optimized device structure, 70 $\mathrm{nm}$-diameter nano-rods locating close to $\mathrm{PC}_{61} \mathrm{BM}$ layer and exciting coupled modes at wavelength of $780 \mathrm{~nm}$ can improve the absorption of perovskite and show double F-P resonance character at $\sim 590 \mathrm{~nm}$ and $\sim 780 \mathrm{~nm}$.

\section{Funding}

Education and Scientific Research Project for Young and Middle-aged Teacher of Fujian (Grant Nos. JAT170324), Promotion Program for Young and Middle-aged Teacher in Science and Technology Research of Huaqiao University (Grant Nos. ZQN-PY508), National Natural Science Foundation of China (NSFC) (Grant Nos. 61404053), Research Project of Huaqiao University (Grant Nos. 13BS419).

\section{References:}

[1] Y. Chen, L. Zhang, Y. Zhang, H. Gao, and H. Yan, "Large-area perovskite solar cells - a review of recent progress and issues," RSC Advances., 2018, Vol. 8, pp. 10489-10508.

[2] M. Grätzel, "The light and shade of perovskite solar cells," Nature Materials., 2014, Vol. 13, pp. 838-842.

[3] H. S. Jung, and N. Park, "Perovskite Solar Cells: From Materials 
to Devices," Small., 2014, Vol. 11, pp. 10-25.

[4] W. S. Yang, B. Park, E. H. Jung, N. J. Jeon, Y. C. Kim, D. U. Lee, ... and S. I. Seok, "Iodide management in formamidinium-lead-halide-based perovskite layers for efficient solar cells," Science., 2017, Vol. 356, pp. 1376-1379.

[5] A. Kojima, K. Teshima, Y. Shirai, and T. Miyasaka, "Organometal Halide Perovskites as Visible-Light Sensitizers for Photovoltaic Cells," Journal of the American Chemical Society., 2009, Vol. 131 pp. 6050-6051.

[6] Q. Q. Lin, A. Armin, R. C. R. Nagiri, P. L. Burn, and P. Meredith, "Electro-optics of perovskite solar cells," Nature Photon., 2014, Vol. 9, pp. 106-112.

[7] C. S. Ponseca, T. J. Savenije, M. Abdellah, K. Zheng, A. Yartsev, T. Pascher, ... and V. Sundström, "Organometal Halide Perovskite Solar Cell Materials Rationalized: Ultrafast Charge Generation, High and Microsecond-Long Balanced Mobilities, and Slow Recombination," Journal of the American Chemical Society., 2014, Vol. 136, pp. 5189-5192.

[8] D. B. Mitzi, "Templating and structural engineering in organic-inorganic perovskites," Journal of the Chemical Society, Dalton Transactions., 2001, pp. 1-12.

[9] S. D. Stranks, G. E. Eperon, G. Grancini, C. Menelaou, M. J. P. Alcocer, T. Leijtens, ... and H. J. Snaith, "Electron-Hole Diffusion Lengths Exceeding 1 Micrometer in an Organometal Trihalide Perovskite Absorber," Science., 2013, Vol. 342, pp. 341-344.

[10] G. Xing, N. Mathews, S. Sun, S. S. Lim, Y. M. Lam, M. Grätzel, ... and T. C. Sum, "Long-Range Balanced Electron- and Hole-Transport Lengths in Organic-Inorganic CH3NH3PbI3," Science., 2013, Vol. 342, pp. 344-347.

[11] Z. He, C. Zhong, S. Su, M. Xu, H. Wu, and Y. Cao, "Enhanced power-conversion efficiency in polymer solar cells using an inverted device structure," Nature Photonics., 2012, Vol. 6, pp. 591-595.

[12] T. S. Sherkar, C. Momblona, L. Gil-Escrig, H. J. Bolink, and L. J. A. Koster, "Improving Perovskite Solar Cells: Insights From a Validated Device Model," Advanced Energy Materials., 2017, Vol. 7, p. 1602432.

[13] L. Yang, F. Cai, Y. Yan, J. Li, D. Liu, A. J. Pearson, and T. Wang, "Conjugated Small Molecule for Efficient Hole Transport in High-Performance p-i-n Type Perovskite Solar Cells," Advanced Functional Materials., 2017, Vol. 27, p. 1702613.

[14] D. Zhao, M. Sexton, H. Park, G. Baure, J. C. Nino, and F. So, "High-Efficiency Solution-Processed Planar Perovskite Solar Cells with a Polymer Hole Transport Layer," Advanced Energy Materials., 2014, Vol. 5, p. 1401855.

[15] C. Momblona, O. Malinkiewicz, C. Carmona, A. Soriano-Portillo, L. Gil-Escrig, E. Bandiello, ... and M. Abdellah, "Efficient methylammonium lead iodide perovskite solar cells with active layers from 300 to $900 \mathrm{~nm}, "$ APL Materials., 2014, Vol 2, pp. 3623-3630.

[16] S. Altazin, L. Stepanova, J. Werner, B. Niesen, C. Ballif, and B. Ruhstaller, "Design of perovskite/crystalline-silicon monolithic tandem solar cells," Optics Express., 2018, Vol. 26, pp. A579-A590.

[17] W. Holzer, A. Penzkofer, H. H. Hörhold, D. Raabe, M. Helbig, and M. Abdellah, "Photo-physical and lasing characterization of an aromatic diamine-xylylene copolymer," Optical Materials., 2000, Vol. 15, pp. 225-235.

[18] H. Hoppe, N. S. Sariciftci, and D. Meissner, "Optical constants of conjugated polymer/fullerene based bulk-heterojunction organic solar cells," Molecular Crystals and Liquid Crystals., 2002, Vol. 385, pp. 113-119.

[19] S. Babar, and J. H. Weaver, "Optical constants of $\mathrm{Cu}, \mathrm{Ag}$, and $\mathrm{Au}$ revisited," Applied Optics., 2015, Vol. 54, pp. 477-481.

[20] P. Schau, K. Frenner, W. Osten, T. Weiss, H. Schweizer, H. Giessen, and L. Fu, "Mode coupling and interaction in a plasmonic microcavity with resonant mirrors," Physical Review B., 2011, Vol.
84, p. 235403.

[21] J. Cui, C. Chen, J. Han, K. Cao, W. J. Zhang, Y. Shen, and M. K. Wang, "Surface Plasmon Resonance Effect in Inverted Perovskite Solar Cells," Adv. Sci., 2016, Vol. 3, p. 1500312.

[22] M. Toma, K. Toma, P. Adam, J. Homola, W. Knoll, and J. Dostálek, "Surface plasmon-coupled emission on plasmonic Bragg gratings," Opt. Express., 2012, Vol. 20, pp. 14042-14053. 